\title{
CFD evaluation of the influence of the parts of a shell and tube heat exchanger on heat transfer
}

\author{
Jiři Frank $^{1^{*}}$, Michal Volf ${ }^{1}$, and Stefan Bajić ${ }^{1}$ \\ ${ }^{1}$ University of West Bohemia, Faculty of Mechanical Engineering, Department of Power System \\ Engineering, Univerzitní 22, Pilsen, Czech Republic
}

\begin{abstract}
This article deals with numerical fluid flow and heat transfer simulations of a shell and tube heat exchanger in which cooling water is heated by hot exhaust fumes. This heat exchanger plays a major role in a cogeneration unit, since it is responsible for the effective use of residual heat. The objective of the simulations is to evaluate the effects of various design changes made to the heat exchanger and their influence on the temperature fields and thus on the overall performance and efficiency of the system. In our analysis we looked at the baffles which cause the cross-current flow of water outside the tubes and at the placement of the gas inlet, i.e., on the distribution of the mass flow rate of the exhaust fumes inside these tubes.
\end{abstract}

\section{Introduction}

Cogeneration of electricity and heat is a very attractive way of using primary sources of energy as it allows for highly efficient conversion. The bottleneck of this approach is obviously that at the place of its production, there must be demand for the heat energy, either for heating purposes or for industrial use. The principle of cogeneration is not new or groundbreaking as there are many power plants that produce both electricity and heat. However, over the last decade this principle is being used in much smaller units, cogeneration units. Their advantage is that they can be placed directly in industrial facilities, residential buildings, and many other places where both electricity and heat are needed. This greatly increases the opportunities for implementing cogeneration.

A cogeneration unit consists of many components: the engine (or generally a device for converting primary energy into mechanical energy, i.e., the rotary motion of a shaft), a generator for converting the mechanical energy into electrical energy and finally a heat exchanger in which the waste heat is transferred into a secondary circuit and used further.

Such a heat exchanger, which plays a major role in the effective use of waste heat, is the focus of our research. We investigate a shell and tube heat exchanger, in which hot exhaust fumes (coming out of an engine) are heating up cooling water flowing in a secondary circuit. The hot water is then further used by a customer. The goal of our research is to evaluate the effects of various design changes made to the heat exchanger and their influence on the temperature fields and thus on the overall performance and efficiency of the system. In our analysis we focus on the baffles which cause the cross-current flow of water outside the tubes

\footnotetext{
* Corresponding author: frankji@students.zcu.cz
} 
and on the placement of the gas inlet, i.e. we investigate the distribution of the mass flow rate of the exhaust fumes inside the tubes.

In the first part of the article, the design of the heat exchanger is briefly described and the main parts which are of interest are discussed in detail. Secondly, the proposed modifications of the geometry, which include changes in the position of the gas inlet and the design of the baffles, are described. Then the simulation setup is discussed. Finally, the simulations are evaluated and we present our results. It should also be noted that all presented simulations were carried out using the Ansys CFX 2019 R1 [1].

Finally, it should be noted that similar studies have also been conducted by various scientists, especially regarding the shape and dimensions of the baffles. One can take a look for example at the following comprehensive studies [2] or [3]. The presented research, however, deals also with the inlet and outlet chambers.

\section{Shell and tube heat exchanger}

A shell and tube heat exchanger (see Fig. 1) is a device used to transfer heat between two separated fluids. It consists of several tubes mounted inside a cylindrical shell. In a flue gas heat exchanger water flows on the outside of the tubes while the exhaust gas flows inside them. Other main parts of the heat exchanger are the inlet and outlet chambers, tubesheets and baffles. Tubesheets are rigidly connected with the tubes and thus determine their exact placement. The inlet chamber is responsible for distributing the exhaust gases to individual tubes.

According to the source [2], the most important geometric parameters are the tube arrangement, the type, the orientation and the opening of the baffles, the space between the baffles. However, we focus mainly on the baffles that are present in the heat exchanger for adequate circulation of working fluid inside the shell [3], It follows that they make a barrier to the flowing water and therefore constrain the direction in which the water flows.

Bear in mind that such a shell and tube heat exchanger consists of many other parts that are not mentioned in the article since they are not relevant for the purposes of our research. [4] [5]

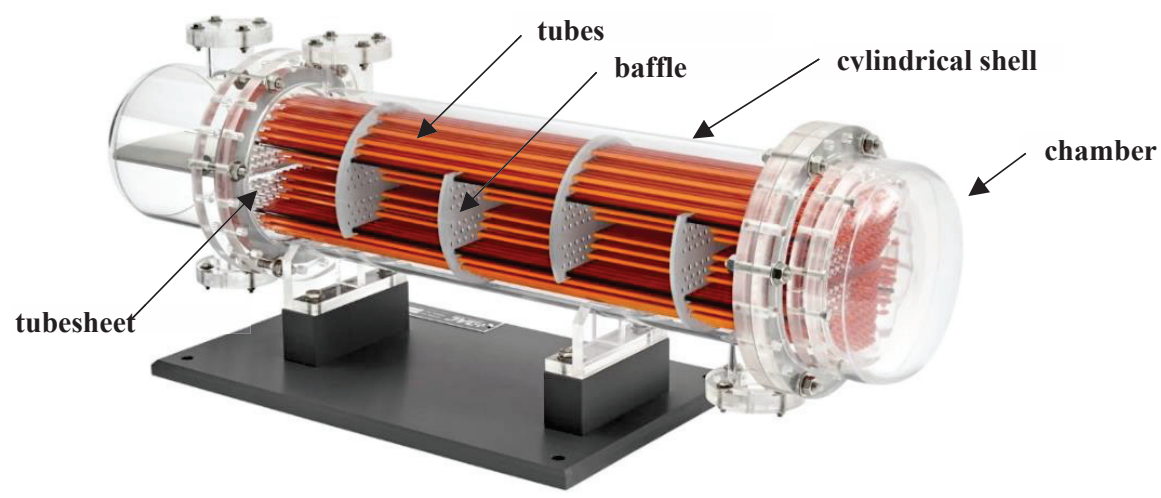

Fig. 1. Shell and tube heat exchanger (generic illustration) [7] 


\section{Geometric modifications of selected parts}

\subsection{Inlet and outlet chambers}

In order to evaluate the influence of the gas chambers, their inlets and outlets were modified. The basic configuration is depicted in Fig. 2 where both gas inlet and outlet are radial.

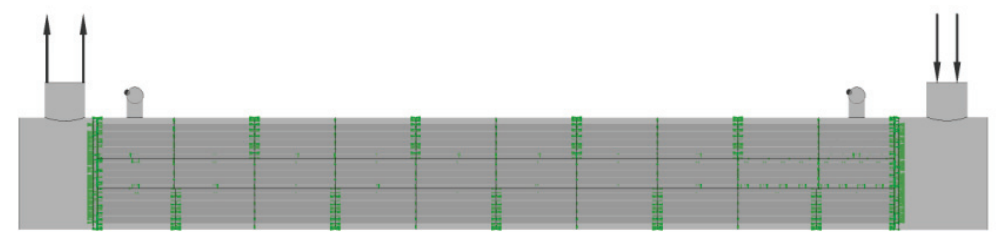

Fig. 2. Basic configuration of gas inlet and outlet

Radial air intake causes the gas in the chamber to whirl and thus the mass flow rate distribution in the tubes is not homogenous as some of the tubes are left outside the main gas flow.

To evaluate the influence of this air whirling in the chambers, several modifications were designed (Fig. 3). The basis of these modifications is changing the radial inlet or outlet to an axial one and their different combinations.
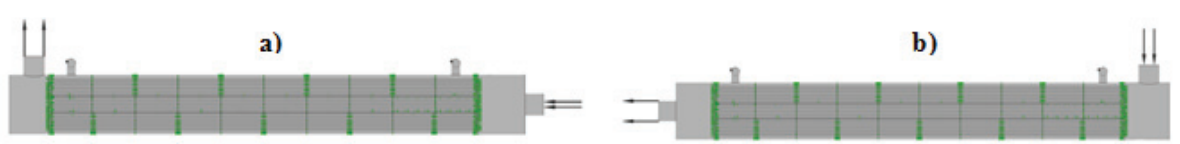

c)

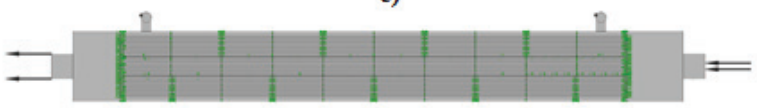

Fig. 3. Proposed modifications to chambers: a: axial inlet \& radial outlet; b: radial inlet \& axial outlet; c: axial inlet \& axial outlet

\subsection{Baffles}

Two parameters were used for optimizing the baffles. First, the length of the baffles was modified to cause changes in the flow area. The idea behind this change is that the lower the flow area, i.e., the greater the length of the baffles, the higher the water velocity. Higher water velocity means that there will be a greater inertia effect causing the water to not so easily change its flow direction. On the other hand, if the flow area is too large, meaning it would not be significantly restricted, there would be no substantial change in the flow direction (i.e., significant reduction in pipe cross-circulation) and thus the effect of the baffles would likely be negligible. It follows that a significant reduction in pipe cross-circulation could even lead to a stoppage of the flow and vortex formation in some areas which would greatly reduce the thermal efficiency of the exchanger. Finally, it should also be noted that a smaller crosssectional area can result in a large increase in pressure drop. Keeping this in mind, two lengths for the baffles were selected. 
In the first variant (Fig. 4a) the baffles go slightly above the plane of symmetry which will prevent the water from flowing purely in the axial direction; however, it will not significantly decrease the flow area and thus will not greatly increase the inertia effect. For the second modification (Fig. 4b), the length of the baffles was increased considerably in order to decrease the flow area and thus to evaluate the effect of inertia. The length of the baffles was selected based on the literature, where it is reported that the most used cut-out height is between $20 \%-25 \%$ of the shell diameter (Fig. 4). Bear in mind that for our analysis we have chosen a value of $20 \%$. Since the base cut-out height was $126 \mathrm{~mm}$, it has been reduced to $69.6 \mathrm{~mm}$.

a)

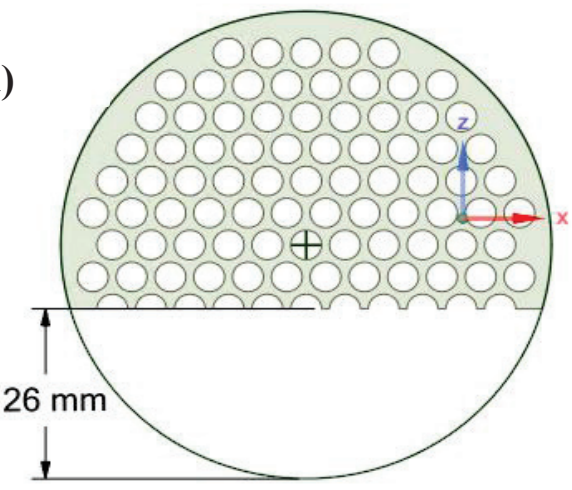

b)

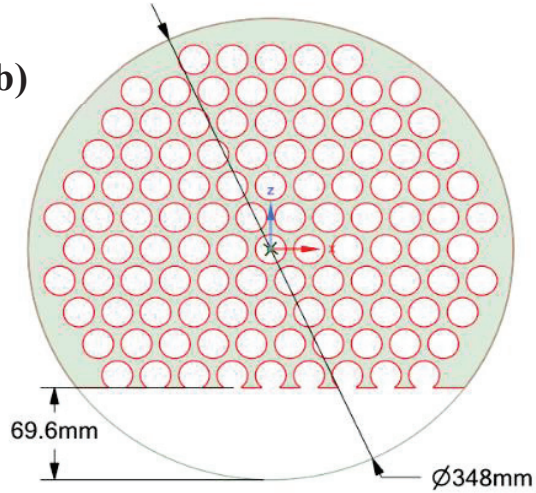

Fig. 4. Modification of the length of baffles

The distance between the baffles was used as the second parameter. Bear in mind that with the changing distance, the number of baffles changes as well. For our analysis we have reduced the base distance and increased the number of baffles from 9 to 13 . This modification can be seen in Fig. 5 .

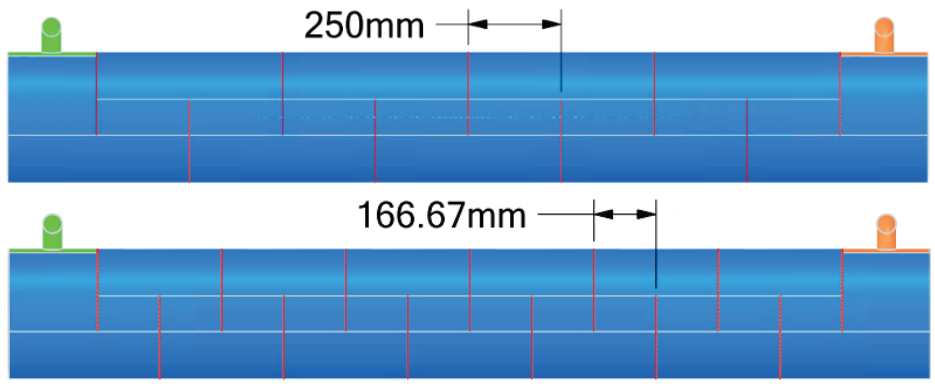

Fig. 5. Modification of distance between baffles

\section{CFD simulations}

The analyses presented in this paper were conducted using the same simulation setup and element sizes of computational meshes, both are discussed in more detail in the following subchapters. Bear in mind that the mathematical model consists of two fluid regions (water and gas) and one solid region (tubes with tubesheets). 


\subsection{Computational meshes}

Computational meshes consist of both tetrahedral and hexahedral elements. Chambers, tubesheets and both input and output pipes for cooling water were discretized using tetrahedral elements in order to locally decrease element sizes and thus to get denser meshes. Other parts were meshed using hexahedral elements. One can see the examples of computational meshes in Fig. 6 and Fig. 7.

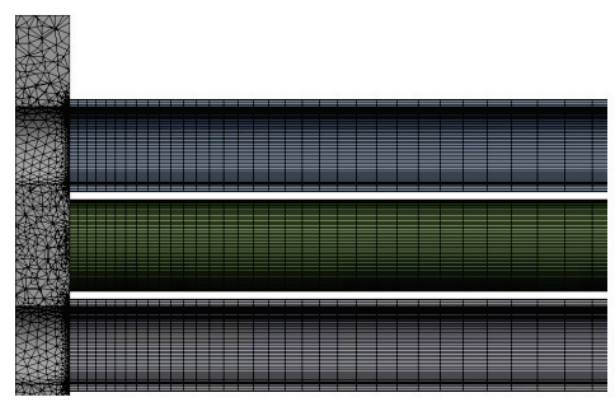

Fig. 6. Mesh for the solid region

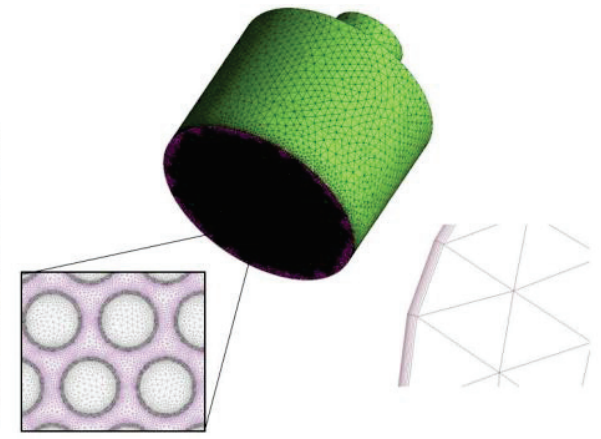

Fig. 7. Mesh of the inlet chamber of the exhaust fumes with a detail of inflation layer

The length of hexahedral elements was chosen to be globally $8 \mathrm{~mm}$ at maximum. Regarding the tubes, the thickness of these elements was $0.5 \mathrm{~mm}$ to get at least three cells across the wall. Element sizes of tetrahedral elements for tubesheets and chambers were $4 \mathrm{~mm}$ and 10 $\mathrm{mm}$ respectively. Concerning the inflation layer, the thickness of the first element was chosen to be $0.05 \mathrm{~mm}$ so that the $\mathrm{Y}^{+}$does not exceed 2.5. In total, the mesh contains approx. 25 million of elements.

For the purposes of the simulation, the baffles were simplified and they were assumed to be of zero thickness, see Fig. 8. Thus, there is no computational mesh prepared for them. This simplifies the model and decreases the number of elements; however, it does not neglect the heat transfer through the baffles because their thickness is defined. Thus, the simulation software (ANSYS CFX) can calculate the heat flux properly.
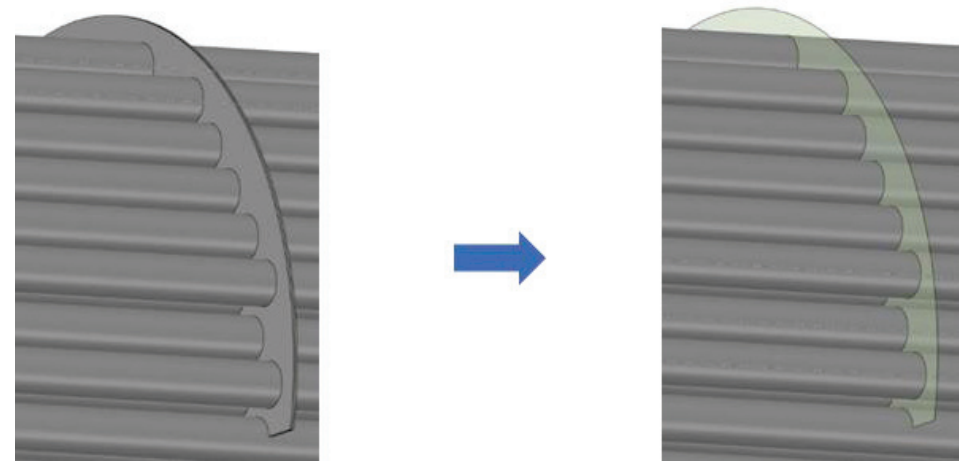

Fig. 8. Simplification of baffles 


\section{Simulation setup}

The presented simulations were done as steady-state ones and thus only boundary conditions were needed, see Table 1 .

Table 1. Boundary conditions

\begin{tabular}{|c|c|c|}
\hline Name & Type of condition & Value \\
\hline \multirow{2}{*}{ Exhaust gas inlet } & Average pressure & $10330[\mathrm{~Pa}]$ \\
\cline { 2 - 3 } & Temperature & $520\left[{ }^{\circ} \mathrm{C}\right]$ \\
\hline Exhaust gas outlet & Mass flow & $0.31\left[\mathrm{~kg} \cdot \mathrm{s}^{-1}\right]$ \\
\hline \multirow{2}{*}{ Water inlet } & Mass flow & $5\left[\mathrm{~kg} \cdot \mathrm{s}^{-1}\right]$ \\
\cline { 2 - 3 } & Temperature & $88\left[{ }^{\circ} \mathrm{C}\right]$ \\
\hline Water outlet & Average pressure & $250000[\mathrm{~Pa}]$ \\
\hline
\end{tabular}

Bear in mind that the pressure boundary conditions are relative to the reference pressure which is set to the atmospheric pressure of $101325 \mathrm{~Pa}$. The exhaust fumes flowing inside the tubes are modelled as an ideal gas with the properties of carbon dioxide, see Table 2 . The water properties are taken from IAPWS and the material properties of X6CrNiTi1810 used for the tubes with tubesheets are given in Table 3.

Table 2. Properties of exhaust fumes (modelled as the ideal gas)

\begin{tabular}{|c|c|}
\hline Property & Value \\
\hline Molar mass & $44.01\left[\mathrm{~kg} \mathrm{kmol}^{-1}\right]$ \\
\hline Specific heat capacity & $851\left[\mathrm{~J} \mathrm{~kg}^{-1} \mathrm{~K}^{-1}\right]$ \\
\hline Thermal conductivity & $0.0145\left[\mathrm{~W} \mathrm{~m}^{-1} \mathrm{~K}^{-1}\right]$ \\
\hline Dynamic viscosity & $1.49 \mathrm{E}-5\left[\mathrm{~kg} \mathrm{~m}^{-1} \mathrm{~s}^{-1}\right]$ \\
\hline Density & ideal gas equation \\
\hline
\end{tabular}

Table 3. Material properties of $\mathrm{X} 6 \mathrm{CrNiTi} 1810$

\begin{tabular}{|c|c|}
\hline Property & Value \\
\hline Density & $8030\left[\mathrm{~kg} \mathrm{~m}^{-3}\right]$ \\
\hline Specific heat capacity & $502.48\left[\mathrm{~J} \mathrm{~kg}^{-1} \mathrm{~K}^{-1}\right]$ \\
\hline Thermal conductivity & $16.27\left[\mathrm{~W} \mathrm{~m}^{-1} \mathrm{~K}^{-1}\right]$ \\
\hline
\end{tabular}

Last but not least, a two-equation SST k- $\omega$ turbulence model is used with a generic turbulence intensity of $5 \%$ at the inlet. Moreover, gravity is considered and it is assumed that the heat exchanger is placed horizontally. All walls are assumed to be hydraulically smooth. All outer walls of the shell and both inlet and outlet gas chambers are adiabatic and thus no heat transfer between the heat exchanger and ambient environment is modelled. 


\section{Evaluation of CFD analysis}

In the following paragraphs, we look at the analysis of the CFD results. First we discuss the influence of the design of the inlet and outlet gas chambers on the performance of the heat exchanger. Then we focus on the evaluation of the baffle design. The contours of the temperatures, mass flow rates and velocities are described.

\subsection{Evaluation of the chamber variants}

Table 4 shows the differences in the input and output values of the monitored parameters for the exhaust gas. It is evident that in the variants with an axial inlet there is less pressure loss than in the variants with a radial inlet. The smallest pressure drop is shown by the variant with both axial inlet and outlet volumes. These changes are not very important for the final temperature of the exhaust gas.

Table 4. Results of modifications to the design of the chambers for the exhaust gas

\begin{tabular}{|c|c|c|c|c|c|}
\hline \multicolumn{7}{|c|}{ EXHAUST GAS } \\
\hline & Base & $\begin{array}{c}\text { Axial inlet } \\
\text { \& radial } \\
\text { outlet }\end{array}$ & $\begin{array}{c}\text { Radial inlet } \\
\text { \& axial } \\
\text { outlet }\end{array}$ & $\begin{array}{c}\text { Axial inlet } \\
\text { \& axial } \\
\text { outlet }\end{array}$ & Units \\
\hline $\begin{array}{c}\text { Pressure difference } \\
\text { Temperature } \\
\text { difference }\end{array}$ & 484.0 & 385.0 & 480.6 & 376.8 & {$[\mathrm{~Pa}]$} \\
\hline
\end{tabular}

The resulting values for cooling water for all the variants are listed in Table 5. As can be seen, changes in the geometry of the inlet and outlet cause differences in pressure losses in the range of $\pm 0.4 \%$. For the cooling water velocity, these differences are approx. $5 \%$. Only in the first new variant will the cooling water heat up significantly, by up to $19 \%$ compared to the original design.

Table 5. Results for modifications to the design of the chambers for cooling water

\begin{tabular}{|c|c|c|c|c|c|}
\hline \multicolumn{7}{|c|}{ COOLING WATER } \\
\hline & Base & $\begin{array}{c}\text { Axial inlet } \\
\text { \& radial } \\
\text { outlet }\end{array}$ & $\begin{array}{c}\text { Radial inlet } \\
\text { \& axial } \\
\text { outlet }\end{array}$ & $\begin{array}{c}\text { Axial inlet } \\
\text { \& axial } \\
\text { outlet }\end{array}$ & Units \\
\hline Pressure difference & 8540.0 & 8570.3 & 8568.8 & 8575.9 & {$[\mathrm{~Pa}]$} \\
\hline $\begin{array}{c}\text { Temperature } \\
\text { difference }\end{array}$ & 3.9 & 4.6 & 4.3 & 4.2 & {$\left[{ }^{\circ} \mathrm{C}\right]$} \\
\hline
\end{tabular}

It can be concluded that the modifications to the chambers do not have too much influence on the resulting flue gas temperature. For the configuration with the axial inlet and radial outlet and the configuration with the radial inlet and axial outlet, the pressure losses in the exhaust gas were reduced due to the axial inlet volume, which causes a more homogeneous distribution of exhaust gases in the tubes. In the axial inlet \& radial outlet configuration, the change to the inlet chamber results in a more uniform heating of the cooling medium. This value is up to $19 \%$ higher than in the basic configuration. This temperature rise is due to a more homogeneous distribution of the exhaust gas in the tubes and a more uniform heating 
of the cooling medium throughout the heat exchanger (see Fig. 9). Thus, the configuration with axial inlet and radial outlet seems to be the most advantageous in terms of thermal energy transfer.

Axial inlet \& radial outlet configuration

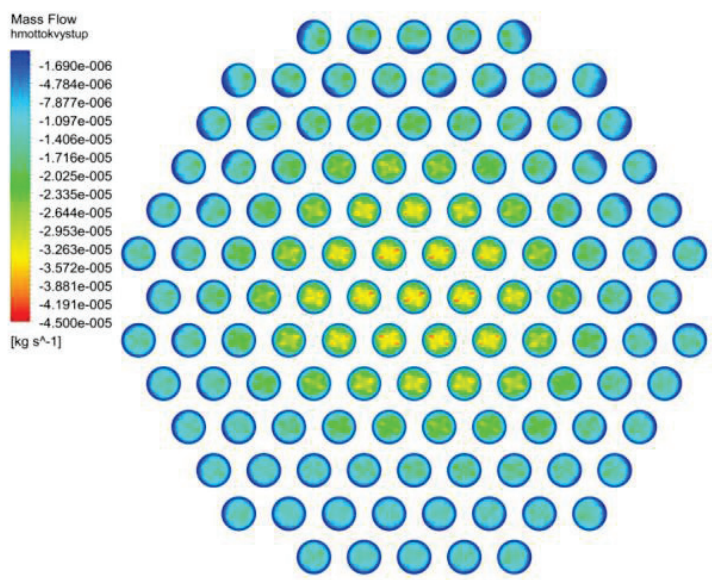

Base

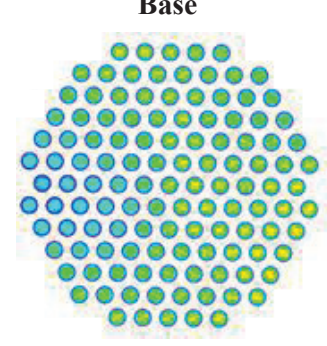

Fig. 9. Mass flow distribution at the outlet from tubes [6]

\subsection{Evaluation of the baffle design}

Fig. 10 shows the velocity contours (left) and the temperature contours (right). All the contours represent the water side of the heat exchanger since this is the main area of interest for these analyses.

It can be seen that in the basic configuration, there is a rather significant region behind the baffles where the flow of water is slowed down and a vortex region is established. This effect is undesirable for heat transfer since water accumulates here which heats up and thus the temperature difference decreases in these regions. The lower the temperature difference, the lower the heat flux and thus the cooling effect is reduced. Thus, we need to modify the geometry of the heat exchanger to get rid of these regions.

The configuration with a smaller distance between the baffles is shown in the middle diagram in Fig. 10. One can see that this configuration has a smaller region of vortices behind the baffles, which is good for the heat transfer. The shorter baffle length in the third variant creates a much greater vortex region, as shown in the last diagram in Fig. 10.

Table 6 and Table 7 sum up the results of these CFD simulations. It is evident that the configuration with the modified distance between the baffles has the highest temperature difference between the inlet and outlet on the water side; however, this improvement is accompanied by increased pressure loss. 


\section{Basic configuration}
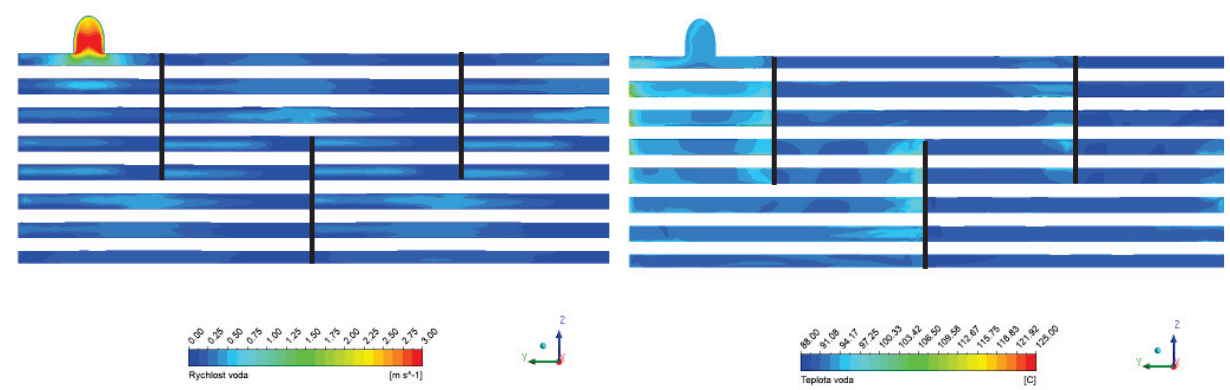

Modification of distance between
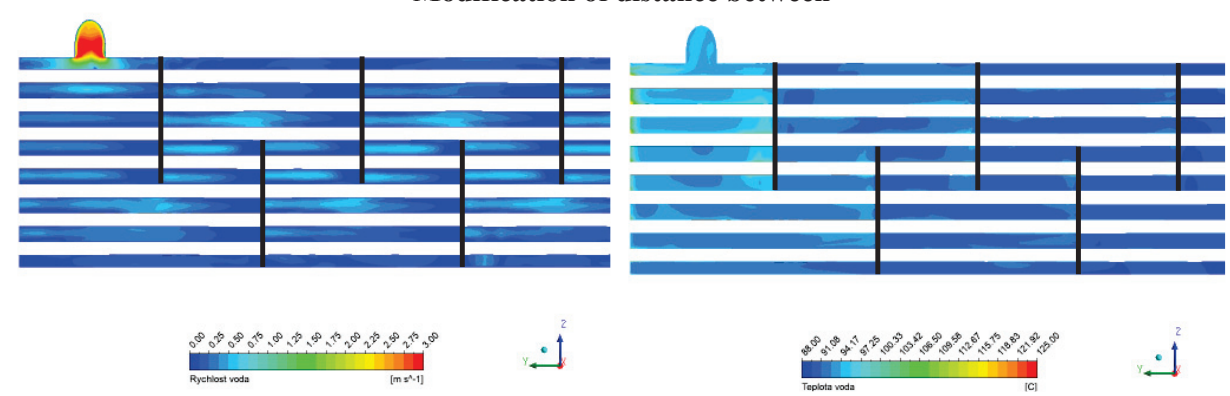

$.1^{2}$

Modification of baffle
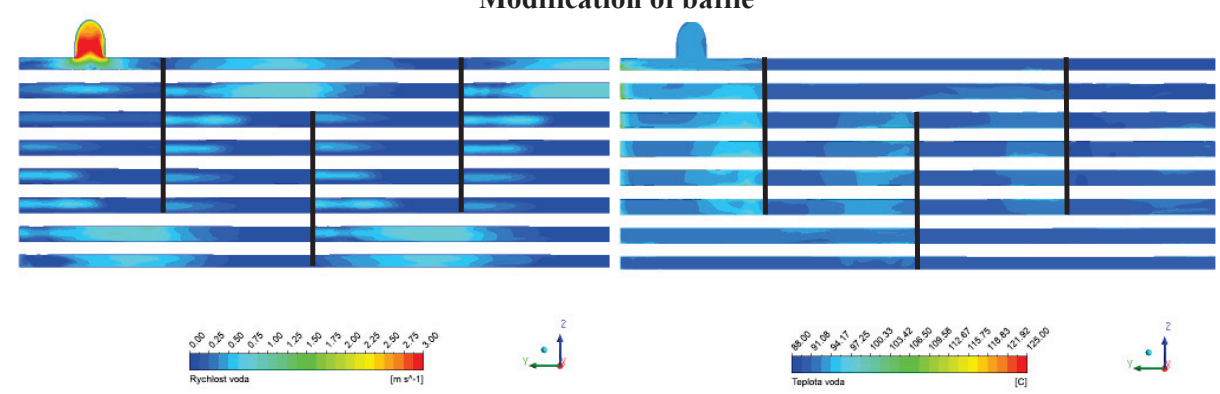

Fig. 10. Current and temperature field of water 
Table 6. Results for modifications to the baffle design for the exhaust gas

\begin{tabular}{|c|c|c|c|c|}
\hline \multicolumn{5}{|c|}{ EXHAUST GAS } \\
\hline & Base & $\begin{array}{c}\text { Modification of } \\
\text { distance between } \\
\text { baffles }\end{array}$ & $\begin{array}{c}\text { Modification of } \\
\text { baffle length }\end{array}$ & Units \\
\hline Pressure difference & 596.0 & 599.0 & 549.0 & {$[\mathrm{~Pa}]$} \\
\hline Temperature difference & 391.1 & 394.9 & 389.5 & {$\left[{ }^{\circ} \mathrm{C}\right]$} \\
\hline
\end{tabular}

Table 7. Results for modifications to the baffle design for the cooling water

\begin{tabular}{|c|c|c|c|c|}
\hline \multicolumn{5}{|c|}{ COOLING WATER } \\
\hline & Base & $\begin{array}{c}\text { Modification of } \\
\text { distance between } \\
\text { baffles }\end{array}$ & $\begin{array}{c}\text { Modification of } \\
\text { baffle length }\end{array}$ & Units \\
\hline Pressure difference & 9179.0 & 11095.0 & 12004.0 & {$[\mathrm{~Pa}]$} \\
\hline Temperature difference & 5.1 & 5.6 & 5.0 & {$\left[{ }^{\circ} \mathrm{C}\right]$} \\
\hline
\end{tabular}

In both modified configurations there is a greater pressure loss on the water side, which is due to the more complicated flow path than in the basic configuration. Regarding the temperature difference, i.e., the heat transfer efficiency, the modification of the distance between the baffles seems to be suitable, as the exhaust gas cools down to a lower temperature and thus transfers more thermal energy to the cooling water (in comparison with the basic modification). From this point of view, it could be concluded that this configuration is better; however, from the point of pressure losses, it is fair to say that decreasing the distance between the baffles results in an increased pressure loss, which is undesirable.

\subsection{Summary of results}

In the following tables, there are summaries of the heat transferred between exhaust fumes and the water. It goes without saying that the higher the value, the better overall design of the exchanger.

Table 8. Summary of heat transferred between exhaust fumes and water (chambers variants)

\begin{tabular}{|c|c|}
\hline Configuration of the heat exchanger & Transferred heat [W] \\
\hline Basic & 81510 \\
\hline Axial inlet \& radial outlet & 96140 \\
\hline Radial inlet \& axial outlet & 89870 \\
\hline Axial inlet \& axial outlet & 87780 \\
\hline
\end{tabular}


Table 9. Summary of heat transferred between exhaust fumes and water (baffle design)

\begin{tabular}{|c|c|}
\hline Configuration of the heat exchanger & Transferred heat [W] \\
\hline Basic & 106590 \\
\hline Modification of distance between baffles & 117040 \\
\hline Modification of baffle length & 104500 \\
\hline
\end{tabular}

To sum up, selected modifications of baffles had slightly positive effect to the value of temperature difference between inlet and outlet of cooling water, but they proved to have quite a large negative effect to the pressure loss in comparison with the base heat exchanger configuration. The pressure loss was higher than $20 \%$ in both modifications and thus it can be concluded that these modifications of baffles are not appropriate.

On the other hand, the modifications in the position of inlet and outlet of chambers seems to result in a significant improvement of the exchanger as, for all modifications, the value of temperature difference between inlet and outlet of cooling water has increased (more than 17 $\%)$. Moreover, the pressure loss of the flowing gas decreased compared with the base variant (more than $20 \%$ ). The configuration with the most significant improvement on the performance of the heat exchanger is the variant with axial inlet and radial outlet, which had the highest temperature difference of cooling water and the lowest pressure loss among all considered configurations.

\section{Conclusion}

This article describes numerical simulations of the exhaust gas and cooling water flow in a shell and tube heat exchanger. The aim of these simulations was to examine the influence of geometric modifications to the inlet and outlet chambers, baffle length and the distance between the baffles on the overall performance of the heat exchanger. Three variants were designed for the inlet and outlet exhaust gas chambers and two variants for the modification of the baffles. These variants were compared with each other and with the basic configuration.

The simulations with modified exhaust gas chambers show that the configuration with the axial inlet and radial outlet seems to be the most advantageous in terms of thermal energy transfer. Regarding the design of the baffles, the simulations show that shortening the distance between them results in better heat transfer; however, it negatively effects the pressure loss on the side of the water. Finally, comparing the modifications to the chambers and baffles, it can be said that the modifications of the inlet and outlet gas chambers have a more positive effect on the heat transfer and thus on the overall heat exchanger performance.

It follows that improvements gained by modifications in inlet and outlet position of chambers could also be beneficial for practical use; however, experimental validation of numerical results (at least partial) must be done.

The presented work was financially supported by the student project SGS-2019-021 (Improving the efficiency, reliability and service life of power machines and equipment 5).

Computational resources were supplied by the project "e-Infrastruktura CZ" (e-INFRA CZ ID:90140) supported by the Ministry of Education, Youth and Sports of the Czech Republic. 


\section{References}

1. Analysis of the influence of parts of a heat exchanger on the current and temperature fields in the heat exchanger using CFD. Bajic, Stefan. 020001, Pilsen : AIP Conference Proceedings, 2019, Vol. 2189.

2. ANSYS®. CFX 2019R1. Release date: January 29th, 2019.

3. Shell Side CFD Analysis of a Model Shell-and-Tube Heat. Máté Petrik, Gábor L. Szepesi. s.1. : AIDIC Servizi S.r.1., 2018, CHEMICAL ENGINEERING TRANSACTIONS.

4. CFD Analysis of a Shell and Tube Heat Exchanger with Single Segmental Baffles. Shuvam Mohanty, Rajesh Arora. 2020, International Journal of Automotive and Mechanical Engineering.

5. Brogan, R.J. Thermopedia. Heat exchangers. [Online] 2 February 2011. [Cited: 6 July 2021.] https://www.thermopedia.com/content/832/.

6. Thulukkanam, Kuppan. Heat exchangers design handbook. 6000 Broken Sound Parkway NW, Suite 300 : Taylor \& Francis Group,, 2013. 13: 978-1-4398-4213-3.

7. Multi-Pass Floating Head Heat Exchanger Training Model. [Online] Technical Laboratory Systems, Inc. [Cited: 236 2021.] https://tech-labs.com/products/multi-passfloating-head-heat-exchanger-training-model. 